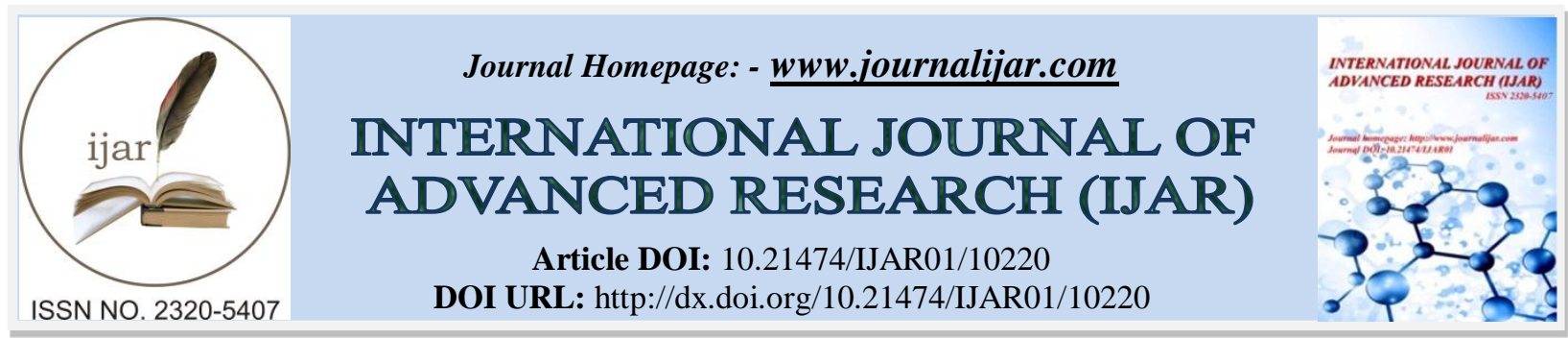

RESEARCH ARTICLE

\title{
CONDUITE A TENIR DEVANT UN MICROPENIS
}

\author{
M. Elbahi, S. Rafi, G. El Mghari and N. El Ansari \\ Service d'Endocrinologie, Diabétologie et Maladies Métaboliques Laboratoire PCIM.FMPM. Chu Mohamed VI, \\ Marrakech.
}

\section{Manuscript Info}

\section{Manuscript History}

Received: 10 October 2019

Final Accepted: 12 November 2019

Published: December 2019

\begin{abstract}
Le micropénis constitue un motif fréquent de consultation en période néonatale, dans l'enfance mais surtout à l'adolescence. C'est un pénis de structure anatomique normale mais d'une longueur $<2,5$ DS en dessous de la moyenne pour l'âge. L'évaluation de la fonction gonadotrope et testiculaire est nécessaire. Elles peuvent être liées à des défauts de sécrétion ou d'action de la testostérone. Il peut être considéré comme une anomalie indépendante ou en tant que résultat de nombreux syndromes congénitaux Indépendamment de la cause sousjacente, un traitement initial par la testostérone en intramusculaire est recommandé pour évaluer la capacité du pénis à y répondre. Le recours à un traitement chirurgical est à considérer si toutes les options du traitement médical sont épuisées. Le retentissement psychologique, particulièrement pénalisant à l'adolescence, doit être pris en compte avec attention. Un suivi est nécessaire par l'endocrinologue, l'urologue, le chirurgien pédiatriques (si la chirurgie est choisie comme traitement).
\end{abstract}

Copy Right, IJAR, 2019,. All rights reserved.

\section{Introduction:-}

Le micropénis est défini comme l'hypoplasie congénitale d'une verge de configuration anatomique normale avec une longueur $<2,5$ DS en dessous de la moyenne pour l'âge [1]. Il constitue un motif fréquent de consultation en période néonatale, dans l'enfance mais surtout à l'adolescence qui est une période de transition vers l'âge adulte et qui se caractérise par une vulnérabilité psychologique.

La fréquence de ce motif de consultation est en augmentation, beaucoup d'étude explique cette augmentation par Une éventuelle contamination parentale par des perturbateurs endocriniens [2,3].

Ces causes sont multiples elles peuvent être liées à des défauts de sécrétion ou d'action de la testostérone. L'altération de l'axe gonadotrope constitue l'étiologie la plus fréquente.

il peut être considéré comme une anomalie indépendante ou en tant que résultat de nombreux syndromes congénitaux. La prise en charge du micropénis reste encore insuffisante alors que des solutions thérapeutiques existent.

\section{Corresponding Author:- M. Elbahi}

Address:- Service d'Endocrinologie, Diabétologie et Maladies Métaboliques Laboratoire PCIM.FMPM.

Chu Mohamed VI, Marrakech. 


\section{Dévelopement normal du pénis: \\ A-In utéro:}

Pendant la vie foetale, le développement normal de la verge commence dès la huitièmesemaine de gestation Sous l'influence de la BHCG placentaire, le testicule foetal produit de la testostérone (T) qui est convertie en dihydrotestostérone (DHT) par le pénis pour en assurer sa différenciation[4]. Cette différenciation est d'ailleurs achevée dès la douzième semaine de gestation. Pendant la deuxième partie de la vie foetale, la croissance du pénis est assurée principalement par les androgènes fœtaux, régulés par la LH fotale. À la quinzième semaine, la verge, y compris le gland et l'orifice urétral, ainsi que le scrotum, sont identifiables [5].

Un pic du taux des androgènes est souvent observé entre la 14éme et le 16éme semaines; entrainantune augmentation marquée de la longueur du pénis au cours des deuxième et troisième trimestres [6].

Ainsi, la différenciation et le développement des organes génitaux externes, notamment de la verge, nécessitent :

1. des testicules normalement différenciés : gènes SRY, SF1, WT1, SoX9...;

2. une production de testostérone normale avec implication des gènes régulant l'action de l'hCg et de la $\mathrm{LH}$, ainsi que des gènes qui gouvernent la stéroïdogénèse testiculaire ;

3. une réceptivité normale aux androgènes qui dépend des gènes de la 5-alpha réductase et du récepteur des androgènes.

\section{En post natal:}

L'activité hormonale de l'axe hypothalamo-hypophysaire et celle des testicules augmente dans les 6 premiers mois de vie postnatale (Figure 1). Une augmentation en volume des testicules et de la longueur du pénis est observée physiologiquement au cours de cette phase active [6].

Pendant cette période, l'hormone folliculostimulante (FSH) et l'hormone lutéinisante (LH) hausse le taux de la testostérone circulante, l'inhibine $\mathrm{B}$ et l'hormone anti-Mullerienne (AMH), parfois même à des niveaux plus élevés que chez les hommes adultes [7].

Il a été constaté que la croissance du pénis atteint son maximum au cours des 3 premiers mois en post natal parallèlement au pic sanguin de la testostérone, son taux diminue à des niveaux pré -pubères au cours des 4-6ème mois suivants [8].

La poussée postnatale des hormones de reproduction apparaît être important pour la croissance des organes génitaux. Un trouble fonctionnel de l'hypophyse ou de l'hypothalamus est responsable d'une croissance inadéquate du pénis en dépit de sa forme normale. De même, un hypogonadisme primaire et par conséquent une production insuffisante de la testostérone en fin de la gestation peut conduire également à un pénis de petite taille.

Il est vraisemblable que des gènes spécifiques de la croissance de la verge, ou des gènes participant à la croissance cellulaire (par exemple du système de l'IgF1), interviennent dans cette régulation [9].

GnRH
hypothalamique

Vie foetale Mini puberté Enfance Puberté et âge adulte



Gonadotrophines circulantes

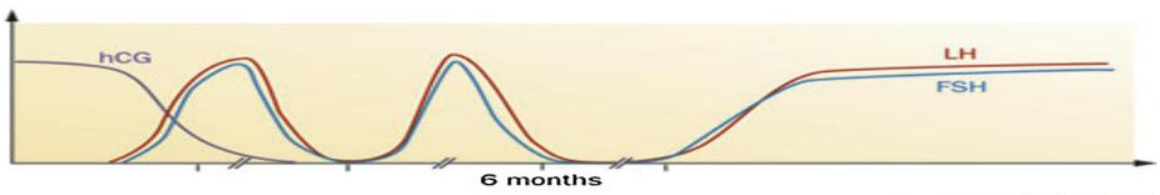

Hormones testiculaires circulantes

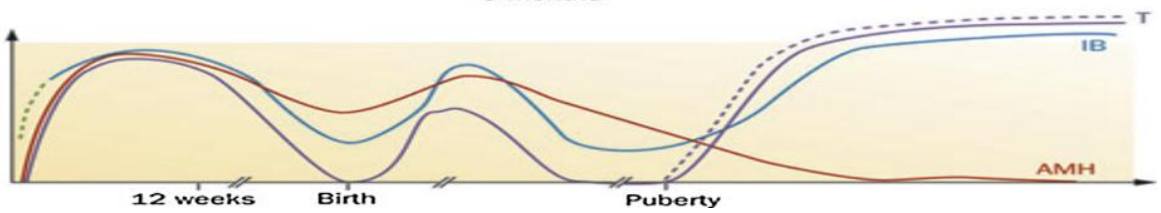

Fig. 1:- Axe gonadotrope et fonction testiculaire au cours de la vie [4]. 


\section{Critères de diagnostic:}

Un diagnostic précoce d'un vrai micropenis. Et donc un examen néonatal complet des organes génitaux externes à la naissance est fort important permettant aux praticiens et aux parents de procéder de façon plus efficace à divers options thérapeutiques.

Une mesure précise qui corrèle avec l'exigence de 2,5 DS en dessous de la norme pour l'âge, avec des organes génitaux externes et internes d'un caryotype XYpeuvent être une preuve suffisante pour soutenir le diagnostic de micropénis.

L'examen clinique doit éliminer une verge enfouie surtout en cas de surpoids, une verge enlisée ou coudée et la présence d'un hypospade.

La mesure de la longueur du pénis se fait sur la face dorsale, de la racine au niveau du pubis, à l'extrémité du gland, en légère traction, sans érection [10].

La verge est saisie entre le pouce et l'index de l'examinateur. La méthode classique utilise une règle ou un étrier pour mesurer la longueur de la verge (Figure 2) [10].

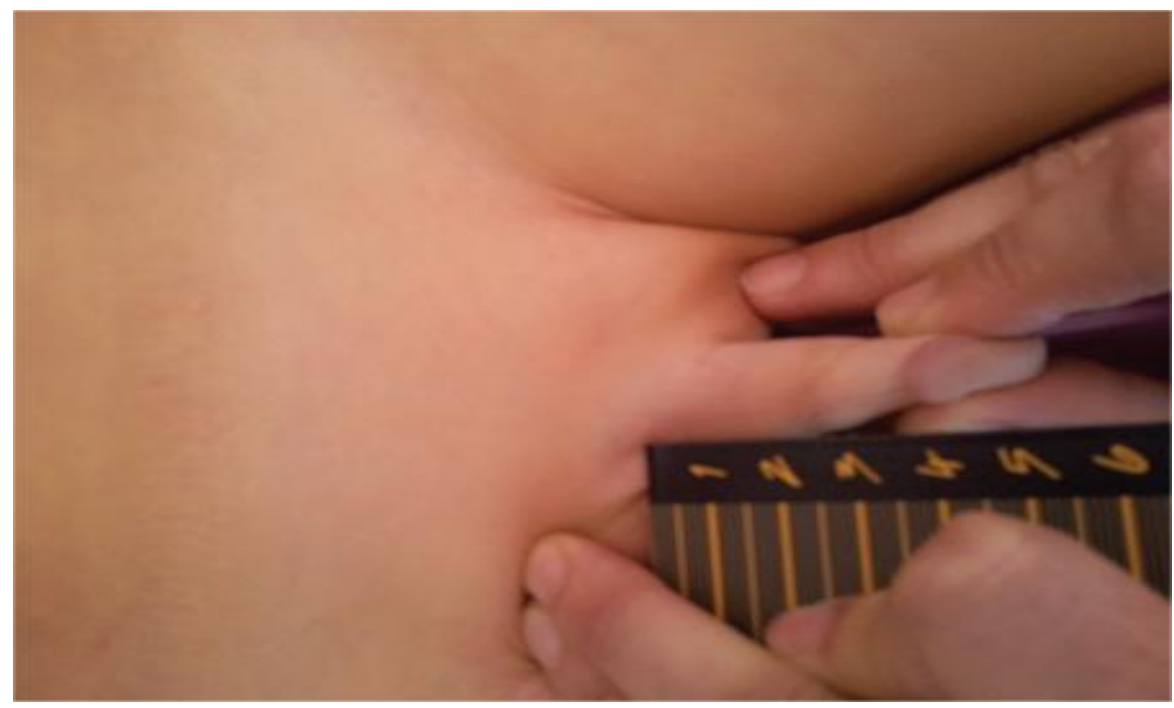

Figure2:- Méthode de mesure de la longueur du pénis.

Dans le cas d'une verge enfouie, il convient d'effectuer une pression sur le tissu adipeux sus-pubien afin de mesurer la longueur dès la racine.

Le diagnostic de micropénis est évoqué si la longueur de la verge est inférieure ou égale à 2,5 DS de la moyenne pour l'âge. Des courbes en fonction de l'ethnie ont été établies. Cheng et al. ont démontré que la longueur et le diamètre moyen du pénis, sont légèrement mais significativement plus faibles chez les nouveau-nés d'origine chinoise que chez ceux d'origine caucasienne ou indienne [11].

La mesure doit être comparée aux valeurs des courbes de référence. La plus connue est la courbe de Schonfeld (Figure 3). La longueur moyenne de la verge des nouveaux nés à terme est de 3,5 $\pm 0,4 \mathrm{~cm}$, et son diamètre de $1,1 \pm$ $0,2 \mathrm{~cm}$. On appelle donc micropénis une verge inférieure à 2,5 DS de la moyenne, schématiquement, de moins de 2 $\mathrm{cm}$ à la naissance, de moins de $4 \mathrm{~cm}$ entre 6 et 12 ans, et de moins de $9 \mathrm{~cm}$ à la puberté La largeur de la verge est importante, l'épaisseur des corps caverneux est souvent faible dans les micropénis [4]. 


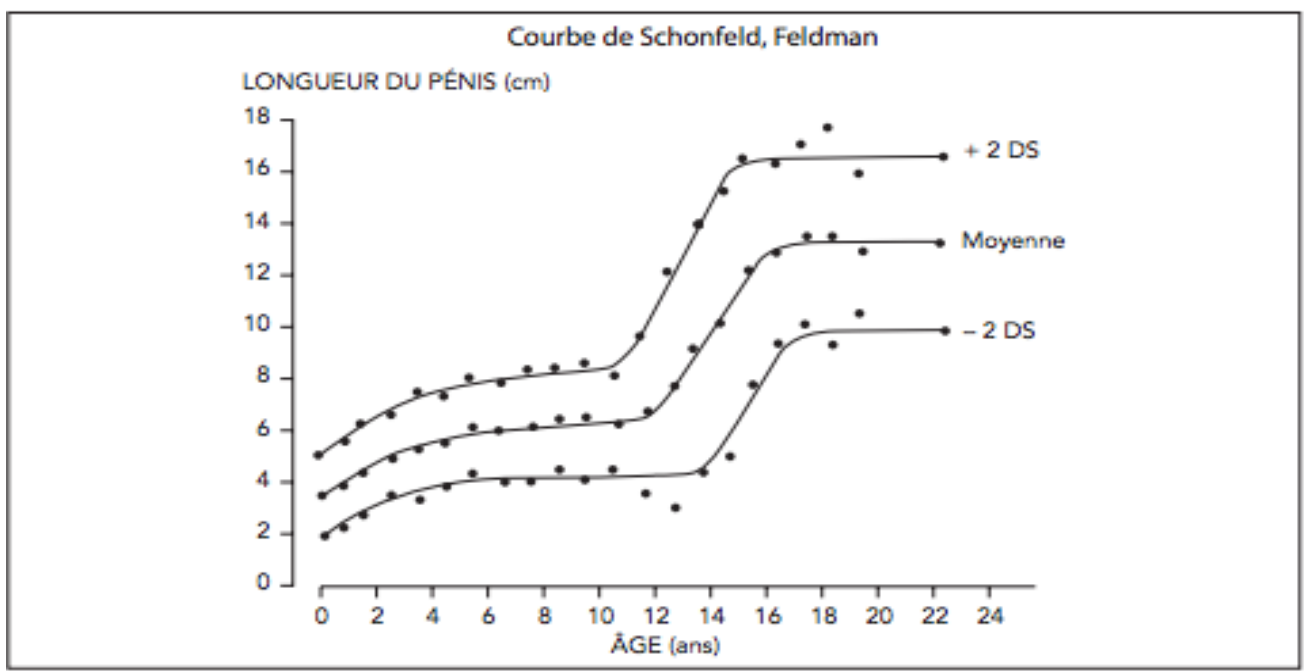

Figure 3:- Taille normale de la verge selon Schonfeld [5].

\section{Quelles sont les causes du micropénis ?}

- Selon les principales données de la littérature [12] , les altérations de l'axe gonadotrope avaient représenté 30 à 40 $\%$ des causes de micropénis ; les hypogonadisme hypogonadotrope secondaires à des anomalies de l'hypophyse ou de l'hypothalamus (le syndrome de Kallman, le déficit gonadotrope isolé ou associé,. . .) ou les hypogonadisme hypergonadotrope secondaires à une anomalie de la biosynthèse de la testostérone et du récepteur LH, un défaut de l'action périphérique des androgènes, une insensibilité partielle aux androgènes ou un déficit en 5 alpha réductase (tableau 1).

Les anomalies génétiques du micropénis (syndrome de Noonan, syndrome de Laurence Moon, Willi Prader, Bardet Biedl ou chromosomiques (syndrome de Klinefelter, syndrome de Down, triploïdies) s'observaient dans $10 \%$ des cas de la littérature[12].

Dans la moitié des cas, l'étiologie du micropénis isolé reste méconnue et il est dit d'origine idiopathique dont la cause est rattachée probablement aux perturbateurs endocriniens environnementaux[2,3].

L'association d'un micropénis à un hypospade, et/ou à une cryptorchidie, est évocatrice d'une anomalie de la différenciation sexuelle XY dont il est fondamental d'identifier la cause.

Tableau 1:- Étiologies du micropénis.

Causes endocriniennes (30-40\%)

$\checkmark$ Hypogonadisme hypogonadotrope

o Isolé (syndrome de Kallman)

o Associé à un syndrome malformatif

o Isolé

$\checkmark$ Déficit somatotrope

o Associé (déficit hypophysaire multiple)

$\checkmark$ Dysgénésie testiculaire

o Régression testiculaire, testicules évanescents

$\checkmark \quad$ Troubles de la synthèse de testostérone

o Anomalies du récepteur de la LH

o Déficits enzymatiques de la stéroïdogénèse

o Déficit en GH/IGF1

$\checkmark$ Résistance aux androgènes

o Déficits en $5 \alpha$-réductase

o Déficit partiel en récepteur des androgènes

Causes génétiques (10\%)

$\checkmark$ Syndromes poly-malformatifs

o Syndrome de Noonan 


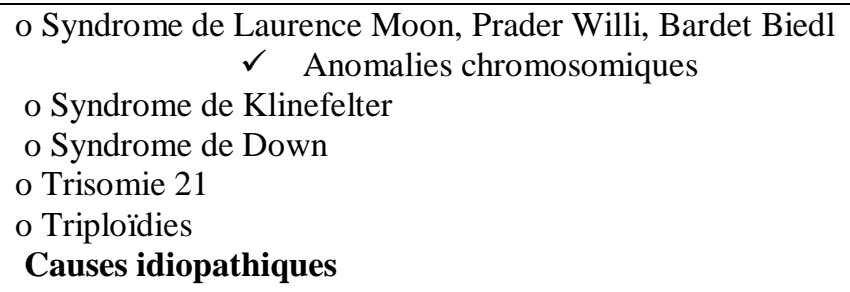

\section{Comment explorer un micropénis ?}

Exploration clinique:

L'Interrogatoire précise la date de constatation pour la lère fois (congénital), les antécédents familiaux (stérilité, retard pubertaire, anomalies des OG), et personnels (hypoglycémie néonatale, anosmie). Un examen génital minutieux permet d'éliminer une cryptorchidie ou un défaut d'implantation du scrotum au dessus de la verge ;

Un examen général doit être pratiqué à la recherche d'un retard de croissance, un syndrome polymalformatif ou dysmorphique et des signes endocriniens (anomalies médio-faciales, des troubles visuels).

\section{Exploration hormonale :}

En période néonatale :

le premier diagnostic à évoquer est celui d'hypopituitarisme congénital. La glycémie doit être mesurée en urgence. Les déficits somatotropes et corticotropes associés entrânent aussi souvent un ictère prolongé qui accompagne les hypoglycémies [4].

Un dosage du taux de cortisol, Insuline-like Growth factor (IGF1) ainsi que celui de la thyroxine libre et totale, déterminera un hypopituitarisme [10].

Un test de stimulation par l'HCG pourrait être fait pour apprécier l'activité leydigienne[4]. Cependant, certains auteurs mettent en avant l'évaluation de la fonction testiculaire par les concentrations plasmatiques d'inhibine B, d'AMH et de testostérone (dans les premiers mois de vie) et ne recommandent pas la réalisation du test à l'hCG [13].

\section{Pendant l'enfance:}

seules les sécrétions Sertoliennes (l'AMH et l'inhibine B) du testicule sont mesurables, la testostérone et les gonadotrophines sont physiologiquement basses[4].

Le dosage de l'AMH est de plus en plus utilisé permettant d'orienter vers une anomalie de la détermination testiculaire en cas de valeur basse [5].

\section{En période pubértaire et peripubertaire :}

-Les niveaux de gonadotrophine sérique (LH et FSH), la testostérone, la DHT et l'androstènedione sont mesurés [10]: Le taux des gonadotrophine sera en mesure d'affinerles diagnostics différentiels, tandis que les niveaux de testostérone et la DHT peuvent déterminer la réactivité des testicules à la stimulation par les gonadotrophines. Ils peuvent également être utilisés pour diagnostiquer un déficit en $5 \alpha$-réductase [10].

-Les Précurseurs de la testostérone : $\Delta 4$-Androstènedione, DHEA, 17 OHP à la recherche de défaut d'enzymes interagissant avec la synthèse de testostérone [10]. -Un test de stimulation par la gonadotrophine chorionique humaine permettra de mesurer la capacité de l'organisme à biosynthétiser la testostérone [10].

\section{Test de stimulation par l'HCG:}

Le dosage de la testostérone après stimulation par l'HCG (1500 UI 1 jour/2 x 7 ou 1000 UI par jour x 3), particulièrement informatif en période néonatale et péri-pubertaire, pour apprécier l'activité leydigienne [5].

En fin de test, l'augmentation de la longueur de la verge traduit une réceptivité normale aux androgènes [14]. Un taux de testostérone inférieur à $300 \mathrm{ng} / \mathrm{dL}$ après stimulation doit faire évoquer une dysgénésie gonadique. 
Si les niveaux de LH et FSH sont élevés, et qu'il n'y a pas d'augmentation du taux de testostérone après stimulation, l'insuffisance ou l'absence de testicules doit être évoquée.

Dans certains cas, un test de sensibilité aux androgènes est nécessaire (Enanthate de testostérone $100 \mathrm{mg} / \mathrm{m}^{2} / 15$ jours $\mathrm{x} 3$ ).

- Dans le cadre du diagnostic d'hypogonadisme hypogonadotrope, l'évaluation de la sécrétion des gonadotrophines par un test de stimulation par la Gonadotrophin releasing hormone $(\mathrm{GnRH})$ peut être réalisé, et lorsqu'elle est effondrée, signe le diagnostic [4].

Les résultats de l'exploration endocrinienne en fonction des différentes étiologies et du stade pubertaire figurent dans le tableau 2 (tableau 2).

Cependant, tout micropénis à testostérone normale, donc considéré alors comme idiopathique, doit faire l'objet d'un interrogatoire sérieux à la recherche de facteurs de risque pendant la vie fœtale (pertubateurs endocriniens).

Tableau 2:- Résultats de l'exploration endocrinienne en fonction des différentes étiologies et du statut pubertaire [14].

\begin{tabular}{|c|c|c|c|}
\hline & $\begin{array}{ll}\begin{array}{l}\text { FSH, } \\
\text { basales }\end{array} & \text { LH }\end{array}$ & $\mathrm{T}$ de base & $\mathrm{T}$ après hCG \\
\hline $\begin{array}{l}\text { Prépuberté } \\
\text { Hypogonadisme hypogonadotrope } \\
\text { Dysgénésie testiculaire } \\
\text { IPA } \\
\text { Idiopathique }\end{array}$ & $\begin{array}{l}\downarrow \\
\mathrm{N} \uparrow \\
\mathrm{N} \\
\mathrm{N} \\
\end{array}$ & $\begin{array}{l}\mathrm{N} \text { Basse } \\
\mathrm{N} \text { basse } \\
\mathrm{N} \\
\mathrm{N}\end{array}$ & $\begin{array}{l}\text { Retardée } \\
\text { Insuffisante } \\
\mathrm{N} \\
\mathrm{N} \\
\end{array}$ \\
\hline $\begin{array}{l}\text { Puberté } \\
\text { Hypogonadisme } \\
\text { Dysgénésie testiculaire } \\
\text { IPA } \\
\text { Idiopathique }\end{array}$ & $\begin{array}{l}\downarrow \\
\uparrow \\
\uparrow \\
\mathrm{N}\end{array}$ & $\begin{array}{l}\downarrow \\
\downarrow \\
\uparrow \\
\mathrm{N}\end{array}$ & $\begin{array}{l}\text { Retardée } \\
\text { Insuffisance } \\
\text { Inutile } \\
\mathrm{N} \\
\end{array}$ \\
\hline
\end{tabular}

T : testostérone ; IPA : Insensibilité Partielle aux Androgènes

Exploration radiologique:

Une échographie pelvienne peut être utile pour apprécier la constitution des organes génitaux internes surtout en cas de pseudohermaphrodisme.

L'Imagerie par Résonnance Magnétique hypothalamo-hypophysaire permet de rechercher une anomalie centrale (une anomalie de la ligne médiane, de la région hypothalamo-hypophysaire et d'apprécier les lobes olfactifs).

\section{Exploration génétique:}

-une analyse du caryotype permet de déterminer le sexe génétique elle est indiqué en présence d'un syndrome dysmorphique, une Bifidité scrotale, un ectopie testiculaire.

\section{Comment traiter ?}

L'objectif du traitement est d'éviter les dysmorphophobies, d'assurer une vie sexuelle normale et des mictions normales.

\section{Traitement médical:}

Les indications et le traitement médicamenteux sont basés sur les recommandations de 2011 de la Société Française d'Endocrinologie pédiatrique [14].

Le traitement médicamenteux du micropénis repose sur l'administration de testostérone. L'objectif est de mimer la sécrétion physiologique de la testostérone en fonction de l'âge, d'éviter les dysmorphophobies, d'assurer une vie sexuelle normale ainsi qu'une miction normale.

\section{Chez le nourrisson:}

La testostérone intramusculaire est efficace. Françoise Paris, comme de nombreux auteurs, préconise un traitement avant l'âge de 2 ans [14]. 
Plusieurs études ont montré que le traitement par testostérone a un effet positif sur la croissance du pénis pendant l'enfance. En effet, il y a une diminution des récepteurs aux androgènes à l'âge adulte, le traitement précoce permet donc d'augmenter la concentration de ces récepteurs $[6,10]$.

De multiples doses et de multiples protocoles existent : trois injections de $50 \mathrm{mg}$ ou 62,5 mg d'Androtardyl (énanthate de testostérone, ampoules $250 \mathrm{mg}$ ), toutes les deux à quatre semaines, ou bien $100 \mathrm{mg} / \mathrm{m} 2$, une injection par mois, pendant trois mois).

Paris et al. proposent, en cas de réalisation de test à l'hCG, avec une réponse inférieure à $3 \mathrm{ng} / \mathrm{ml}$, sans modification de longueur de la verge, de réaliser trois injections d'Enanthate de testostérone, $50 \mathrm{mg}$ toutes les deux semaines. Si la longueur de la verge ne se normalise pas $(>3 \mathrm{~cm})$, il y a lieu de répéter une fois le traitement [14].

-Si le diagnostic d'hypogonadisme hypogonadotrope est confirmé, un traitement par les gonadotrophines recombinantes en sous-cutané peut être discuté. La DHT locale, en gel, est efficace.

\section{Pendant l'enfance:}

Durant cette période, la testostérone de base est ininterprétable, le test à l'hCG est peu informatif et la réponse de la verge aux androgènes moins marquée qu'en période néonatale ou péri-pubertaire. L’androgénothérapie est donc plus difficile à mettre en route.

Toutefois, un micropénis sévère mal toléré doit être traité par testostérone intramusculaire oudihydrotestostérone (Andractim gel 2,5\%:0,3 mg/kg) [16].

\section{En période péri-pubertaire:}

Le traitement s'impose car la puberté est achevée, l'androgénothérapie devient inefficace sur la taille de la verge. A cet âge, l'injection de $100 \mathrm{mg} / \mathrm{m}^{2} / \mathrm{mois}$ d'énanthate de testostérone à trois reprises est habituellement efficace [17]. Durant le traitement, la surveillance de l'évolution de la maturation osseuse et de la croissance est primordiale car celui-ci peut entraîner une accélération de la croissance et une avance transitoire de l'âge osseux.

\section{Traitement chirurgical:}

Une reconstruction chirurgicale du pénis ou l'élongation par chirurgie plastique est une option thérapeutique à tenter après échec d'un traitement médical bien conduit. Elle est rarement effectuée chez l'enfant. Ces procédures sont très complexes, ils peuvent avoir plusieurs complications telles que les cicatrices inesthétiques, la douleur, et une diminution du plaisir sexuel. C'est un geste qui exige une équipe médicale qualifiée, comprenant des chirurgiens plastiques, des chirurgiens microvasculaires, et des urologues [10]. Actuellement ils ne sont effectués que dans des centres hautement spécialisés qui peuvent garantir les meilleurs résultats.

\section{Psychothérapie:}

Dans tous les cas, le conseil psychologique est utile et très probablement nécessaire tant pour l'enfant que les parents et devrait être commencé tôt devant la souffrance émotionnelle ressentie par ces patients. Une Psychothérapie peut être nécessaire à mesure que l'enfant grandit avec sa condition. La peur du rejet sexuel, l'insuffisance sexuelle, la taille de leur pénis, la mauvaise image corporelle du soi, l'altération de la qualité de vie étant les problèmes courants chez ces patients en phase d'adolescence et à l'âge adulte [18].

\section{Conclusion:-}

Le retard à la consultation rend tardive la prise en charge du micropénis dans notre contexte. Le praticien doit, en premier lieu, évaluer l'existence du micropénis avant d'envisager les explorations endocriniennes permettant d'en déterminer l'étiologie.

Chez le nourrisson, un micropénis isolé peut rarement révéler une anomalie de la différenciation sexuelle. À l'adolescence, les rapports sexuels plus précoces et l'accessibilité facile à l'internet peuvent générer de véritables dysmorphophobies.

La prise en charge implique un traitement médical ou chirurgical, un suivi régulier tout au long de l'enfance doit être assuré par un endocrinologue et un psychologue si besoin. 


\section{Bibliographie:-}

1. Wisniewski AB, Migeon CJ, Gearhart JP, et al. Congenital micropenis: long-term medical surgical and psychosexual follow-up of individuals raised male or female. HormRes 2001;56:3-11.

2. Bhangoo A, Paris F, Philibert P, et al. Isolated micropenis reveals partial androgen insensitivity syndrome confirmed by molecular analysis. Asian J Androl 2010;12(4):561 - 6.

3. 3- Paris F, De Ferran K, Bhangoo A, et al. Isolated idiopathic micropenis: hidden genetic defects? Int J Androl 2011;34(6 Pt 2): e518-25.

4. Bouvatier C, Micropénis. ArchPediatr 2014;21:665-9.

5. Paris F, GaspariL., Dutertre E., Philibert P, Maimoun L., Kalfa N, \& Sultan, C. MICROPÉNIS: CONDUITE A TENIR.

6. Hatipoğlu N, Kurtoğlu S. Micropenis: etiology, diagnosis and treatment approaches. J Clin Res PediatrEndocrinol 2013;5:217-23.

7. Grumbach MM. A window of opportunity: the diagnosis of gonadotropin deficiency in the male infant. J ClinEndocrinolMetab. 2005;90:3122-3127.

8. Byne W. Developmental endocrine influences on gender identity: implications for management of disorders of sex development. Mt Sinai J Med. 2006;73:950-959.

9. Levy J.B., D.A. Hussmann :Micropenis secondary to growth hormone deficiency : does treatment with growth hormone alone result in adequate penile growth ? J Urol 1996, 156 : 214-216.

10. 10-Tsang S. When Size Matters : A Clinical Review of Pathological Micropenis. J PediatrHealt Care 2010;4:231-40..

11. Cheng PK, Chanoine JP. Should the definition of micropenis vary according to ethnicity? HormRes 2001;55:278-81. C'était 10

12. Menon PS, Khatwa UA. The child with micropenis. Indian J Pediatr 2000;67(6):455-60

13. Brauner R. Micropenis : quand y penser et que faire ? Real Pediatr 2013;177:12-5.

14. Paris F, Gaspari L, Dutertre E, et al. Micropenis : diagnosis and management. Mises au point cliniques d'endocrinologie 2011;103-8.

15. Bougnères $\mathrm{P}$, François $\mathrm{M}$, Pantalone L, et al. Effects of an early postnatal treatment of hypogonadotropic hypogonadism with a continuous subcutaneous infusion of recombinant follicle-stimulating hormone and luteinizing hormone. J Clin Endocrinol Metab 2008;93:2202-5.

16. Ishii T, Hayashi M, Suwanai A, et al. The effect of intramuscular testosterone Enanthate treatment on stretched penile length in prepubertal boys with hypospadias. Urology 2010;76(1):97-100.

17. Husmann DA, Cain MP. Microphallus: eventual phallic size is dependent on the timing of androgen administration. J Urol 1994;152:734-9.

18. Husmann, D. A. "The androgen insensitive micropenis: long-term follow-up into adulthood." Journal of Pediatric Endocrinology and Metabolism 17 (2004): 1037-1042. 